\title{
Ultrathin and Simple Frequency Selective Rasorber Based on Ferrite Absorber with Embedded Epsilon-Near-Zero Tunneling Channels
}

This paper was downloaded from TechRxiv (https://www.techrxiv.org).

\section{LICENSE}

CC BY 4.0

SUBMISSION DATE / POSTED DATE

28-11-2021 / 07-12-2021

\section{CITATION}

Zhong, Shuomin; Feng, Jiaqi; Zheng, Zi-Wei; Ma, Yungui (2021): Ultrathin and Simple Frequency Selective Rasorber Based on Ferrite Absorber with Embedded Epsilon-Near-Zero Tunneling Channels. TechRxiv. Preprint. https://doi.org/10.36227/techrxiv.17088578.v1

\section{$\mathrm{DOI}$}




\title{
Ultrathin and Simple Frequency Selective Rasorber Based on Ferrite Absorber with Embedded Epsilon-Near-Zero Tunneling Channels
}

\author{
Shuomin Zhong, Jiaqi Feng, Zi-Wei Zheng, Yungui Ma
}

\begin{abstract}
An ultrathin and simple frequency-selective rasorber (FSR) with a passband located within a wide absorption band is proposed. The ultrawide absorption band is obtained by employing commercial magnetic materials in the absorption channel and the passband is realized using epsilon-near-zero (ENZ) tunneling waveguides. The attractively ultrathin and simple feature is achieved by utilizing tunneling effect at the cutoff frequency of metallic waveguides with arbitrary length, permitting the overall thickness shrink into to the same as that of the absorber. A prototype with a thickness of only $0.108 \lambda_{c}\left(\lambda_{c}\right.$ is the free-space wavelength at the transmission frequency) is fabricated and measured, exhibiting a transmission band with -3-dB fractional bandwidth (FBW) of $35.7 \%$ and a minimum insertion loss of $1 \mathrm{~dB}$, and an FBW of $135.6 \%$ for reflection coefficient less than $-10 \mathrm{~dB}$. To demonstrate the main advantages of ENZ-based rasorber in thickness over previous designs further, a rasorber with a record ultrathin thickness of $0.022 \lambda_{c}$ constructed by $\mathrm{SN}-20$ ferrites and $\mathrm{ENZ}$ waveguides is realized numerically. Moreover, the ENZ-based rasorber concept can be extended into both dual-polarized and dual-band designs with remarkable structural simplicity. The lumped-components-free and lithography-free features greatly facilitate the fabrication, which is promising in practical low-cost applications.
\end{abstract}

Index Terms - epsilon-near-zero (ENZ), ferrite absorber, frequency selective rasorber (FSR), ultrathin

\section{INTRODUCTION}

Over the past decade, frequency-selective rasorbers (FSRs) [1-3] have aroused extensive interests aiming for improving stealth performance of radar radomes. Compared with conventional frequency selective surfaces (FSSs), FSRs require high absorption for out-of-band electromagnetic (EM) waves instead of reflection, achieving both mono- and bi-static radar-cross-section (RCS) reduction. Meanwhile, a transmission window with high transmission efficiency for in-band EM waves is another important feature to ensure good communication performance.

Manuscript received September 6, 2021. This work was supported in part by National Natural Science Foundations of China (NSFC) (61701268). (Corresponding author: Shuomin Zhong.)

Shuomin Zhong, Jiaqi Feng are with the School of Information Science and Engineering, Ningbo University, Ningbo 315211, China. (email: zhongshuomin@nbu.edu.cn)

Zi-Wei Zheng is with Digital Industry Research Institute, Zhejiang Wanli University, Ningbo 315211, China.

Yungui $\mathrm{Ma}$ is with the State Key Laboratory of Modern Optical Instrumentations, Centre for Optical and Electromagnetic Research, Zhejiang University, Hangzhou 310058, China.
Conventional two-dimensional (2-D) FSRs [4]-[13] are constructed by cascading a lossless bandpass FSS with a resistor-loaded lossy FSS layer. Since the incident waves have to pass through the lossy layer, 2D designs usually suffer from large insertion loss in transmission band [9], [13]. To address this issue, series $L-C$ circuits are employed to short-circuit the lossy components [4] or parallel $L-C$ resonators [5-12] with intrinsic infinite impedance at resonance are designed to bypass the absorptive parts to achieve high in-band transmission. Nevertheless, it is difficult to construct different resonators for absorption and transmission bands respectively in a single 2-D planar structure, making the 2-D method less flexible.

In these years, new types of three-dimensional (3-D) FSRs [14]-[16] have been proposed, where the absorption and transmission paths are separately constructed, achieving superior performance of low insertion loss and high flexibility. In [16], by loading a capacitor inside the absorptive structure, an absorptive frequency-selective reflection (AFSR) structure is achieved as an absorption path and combined with a metallic-via-loaded parallel-plate waveguide (PPW) as a transmission path. In the transmission band, the wave can bypass the absorption path and transmit through the waveguide with a low insertion loss $(0.43 \mathrm{~dB})$. This 3 -D scheme shows great design flexibility because the absorption and transmission performance are almost independently determined by the AFSR structure and the transmission PPW.

Most of the previous 3-D rasorbers utilize lumped components, including lumped resistors and capacitances, which significantly increase the cost and fabrication complexity. Recently, some commercial magnetic absorbers are employed to construct rasorbers featuring low cost and easy fabrication [17]-[23]. More importantly, the ferrite materials [22] can achieve wide absorption band with ultrathin thickness, greatly enhancing the freedom of absorption path design in the FSR. Then the overall thickness of rasorber is limited by the thickness of transmission path. The thickness of commonly used PPW transmission structure is determined by $t \approx$ $\lambda_{c} /\left(2 \sqrt{\varepsilon_{r}}\right)$, where $\lambda_{\mathrm{c}}$ is the free-space wavelength at transmission frequency, and $\varepsilon_{r}$ represents the relative permittivity of the substrate. In [20], by utilizing a dielectric-filled PPW, the thickness of the FSR is designed as $\lambda_{\mathrm{c}} / 4$. To further reduce the thickness, a slow wave structure with thickness of about $\lambda_{c} / 11$ is proposed in [21]. However, its thickness is still four times larger than the absorption path. Besides, the folding profile in the structure is too complicated and difficult to fabricate. Therefore, designing a transmission structure with ultrathin and simple configuration is vital to fully expose the advantages of magnetic absorbing materials, reducing the overall thickness and complexity of FSR. In this 
paper, by combining a thin wideband ferrite absorber and rectangular metallic waveguides with tunneling effect at cutoff frequencies [24]-[27], an ultrathin and simple 3-D FSR is proposed.

The major contributions of this work are as follows.

1. The magnetic absorbing materials are tailored into an absorptive frequency-selective reflection (AFSR) structure by loading a dielectric layer, with a specific reflection band within an ultrawide absorption band, averting the use of band-stop FSS to bypass losses in the absorption path. A low-insertion-loss transmission window within an ultrawide absorption band is obtained in lumped-components-free and lithography-free structure, featuring low cost and easy fabrication.

2. The simple rectangular metallic waveguides mimicking zero-permittivity properties at the cutoff frequency, with ENZ tunneling effect, are first utilized to construct transmission path in 3-D FSRs. Superior to conventional PPW requiring length of half a guided wavelength, the ENZ waveguide can transmit the wave near the cutoff frequency, with arbitrarily short propagating length, due to the phase invariance along the channel. Therefore, the length of transmission path will no longer be a limit for the ultimate thickness of FSR, which can be designed as thin as the ultrathin ferrite absorber. An extremely thin ENZ rasorber working in the same band with the one in [21] is proposed further. By replacing the bulk slow-wave structure into the ENZ waveguides, the thickness is reduced dramatically from $0.086 \lambda_{\mathrm{c}}$ to 0.022 $\lambda_{c}$, with almost the same transmission and absorption performance maintained.

3. The weak coupling between different tunneling channels, give the ENZ waveguide frequency-selective transmission structures great flexibility in achieving dual-polarized and multi-band FSRs in quite simple configurations.

The remaining of this article is organized as follows. In section II, the simple rasorber is described in detail. Then the operating principles of the ferrite absorber and the ENZ tunneling waveguide are analyzed. Measurement results are introduced in section III. In section IV, several designs inspired by ENZ tunneling effect are proposed, demonstrating great advantages in implementing extremely-thin, dual-polarized and dual-passband FSRs in a simple way. Finally, a conclusion is given in Section V.

\section{Design PRINCIPLE}

\section{A. Description of the Structure}

The configuration of the 3-D FSR proposed in this paper is shown in Fig. 1. The electric field is polarized along the $y$-direction, the magnetic field is along the $x$-direction, and the wave propagates along $-z$-direction (normal incidence). Each unit cell consists of two parts, including the upper absorption part and the under transmission part. The transmission channel is a metallic rectangular waveguide, with a height $h_{2}$, a width $w$ and a length $t$. The upper absorption channel consists of two different layers with thickness $t_{1}$ and $t_{2}$, respectively. The height of the ferrite material is $h_{1}$ and a metallic plate with the same height is at the back of the ferrites. The thickness of the whole

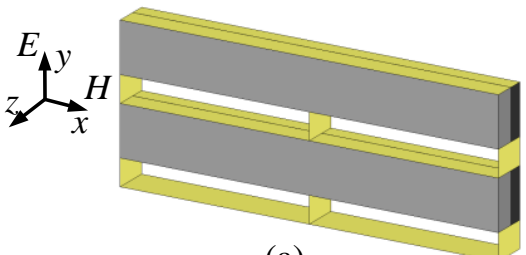

(a)

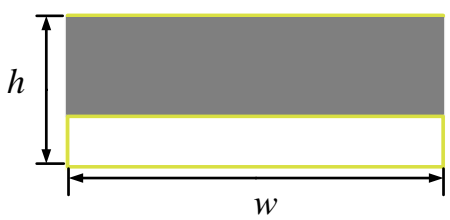

(c)

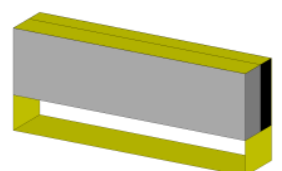

(b)

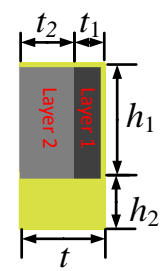

(d)
Fig. 1. Structure of the proposed FSR. 3-D views of (a) $2 \times 2$ unit cells and (b) a unit cell for concept illustration. (c) Front and (d) side views of a unit cell. (Physical dimensions: $h=13 \mathrm{~mm}, h_{1}=8.5 \mathrm{~mm}, w=32 \mathrm{~mm}, h_{2}=4.5 \mathrm{~mm}, t=6.3 \mathrm{~mm}$, $t_{1}=2.6 \mathrm{~mm}, t_{2}=3.7 \mathrm{~mm}$ ). Yellow denotes metal, grey denotes dielectric layer 2 , and black denotes ferrite layer 1 .

structure along the $z$-direction is $t=t_{1}+t_{2}$. The periods of the unit cell are $w$ along the $x$-direction and $h=h_{1}+h_{2}$ along the $y$-direction. In simulation, a unit-cell boundary condition is set along the $x$ - and $y$-directions, and the number of Floquet modes is set as 8 to include all the possible propagating modes with high orders in the high frequency bands.

\section{B. Absorption Path Analysis}

The absorption path is accomplished by double-layer absorbing materials with a metallic plate on the back. The absorber used in our rasorber is made of LCXJXB rubber ferrite absorbing materials from Zhejiang Luchuang Material Technology Co., Ltd., China, as shown in Fig. 2(a), consisting of two different flexible layers. The complex relative permittivity $\left(\varepsilon_{\mathrm{r}}=\varepsilon_{\mathrm{r}}{ }^{\prime}-j \varepsilon_{\mathrm{r}}{ }^{\prime \prime}\right)$ and permeability $\left(\mu_{\mathrm{r}}=\mu_{\mathrm{r}}^{\prime}-j \mu_{\mathrm{r}}{ }^{\prime \prime}\right)$ of the two layers are plotted in Fig. 2(b). It is seen that, for layer 1, its permittivity and permeability show strong frequency dispersion with both high magnetic and dielectric loss. The real part of permittivity of layer 1 is around 20 in the whole band interested, and the real part of permeability decreases from 4 to 1.1 with frequency from 2 to $18 \mathrm{GHz}$. Different from layer 1 with high dispersion and loss, the layer 2 is a rubber with permittivity of $\varepsilon_{\mathrm{r} 2}{ }^{\prime}=2.6$, permeability of $\mu_{\mathrm{r} 2}{ }^{\prime}=1.1$, and low lossy part $\left(\varepsilon_{\mathrm{r} 2}{ }^{\prime \prime}=0.12\right.$, $\left.\mu_{\mathrm{r} 2}{ }^{\prime \prime}=0.05\right)$.

To demonstrate the role of layer 2 in the absorption path design, the reflections of the magnetic absorbers with layer 2 and without layer 2 are simulated and compared. As shown in Fig. 2(c), with layer 1 only, the absorber exhibits a high-absorption band from 2.3 to $4.3 \mathrm{GHz}\left(\left|S_{11}\right|<-10 \mathrm{~dB}\right)$, and a flat and wide reflection band with $\left|S_{11}\right| \approx-5 \mathrm{~dB}$ (75\% absorption) from 6 to $18 \mathrm{GHz}$. This property is attributed to the low permeability in the high band, resulting in impedance mismatch. Therefore, to improve the absorption performance in high band, the layer 2 with a thickness of $3.7 \mathrm{~mm}$ is introduced. It can be seen in Fig. 2(c) that with layer 2, the reflection in high band is suppressed largely below $-10 \mathrm{~dB}$ from 8.7 to $18 \mathrm{GHz}$. The mechanism can be explained from the input impedances of the absorbers with layer 2 and without layer 2. In Fig. 2(d), with layer 1 only, the input impedance has a flat value of about $116-j 20 \Omega$ in the high band, which is not well-matched to the 
free space impedance. By adding layer 2, the input impedance is transformed into a well-matched value of $437 \Omega$ at $13 \mathrm{GHz}$, corresponding to a reflection dip lower than $-20 \mathrm{~dB}$. Thus, the layer 2 plays a role of quarter-wave matching transformer, improving the absorption performance in high band. In the low absorption band, the perfect impedance matching frequency shifts from $3.3 \mathrm{GHz}$ to $2.8 \mathrm{GHz}$ when the layer 2 is considered. Additionally, there is a reflection peak of $-5.5 \mathrm{~dB}$ at $5.45 \mathrm{GHz}$. This absorptive frequency-selective reflection property is beneficial for the absorption path design of rasorber, to bypass the losses in the passband when it is located in the frequency-selective reflection band.

In our 3-D FSR design, the magnetic absorber is filled into a parallel plate waveguide (PPW) [see Fig. 1]. As indicated in [20], the EM field distribution will not be affected by the PPW. Therefore, the reflection coefficients should be identical for the grounded magnetic materials and the short-circuited magnetic-materials-filled PPW. However, when the parallel metallic plate has a certain thickness, the metallic block will disturb the original EM field, leading to unavoidable influence to the reflection characteristic. Similar with previous studies [20], we replace the transmission channel with a PEC block with the identical size, as depicted in Fig. 3(a). The equivalent circuit model (ECM) of the absorption channel with PEC block is presented in Fig. 3(b). The port impedance can be written as $Z_{0 f s r}=\eta_{0}\left(h_{1}+h_{2}\right) / w$, which is related to the heights of magnetic material and PEC block. The characteristic impedances and complex propagation constants of the PPWs filled with layers 1 and 2 are

$$
\begin{gathered}
Z_{m 1}=\eta_{0} \frac{h_{1}}{w} \sqrt{\frac{\mu_{\mathrm{r} 1}}{\varepsilon_{r 1}}}, \\
k_{m 1}=\omega \sqrt{\mu_{0} \varepsilon_{0}} \sqrt{\mu_{r 1} \varepsilon_{r 1}}, \\
Z_{m 2}=\eta_{0} \frac{h_{1}}{w} \sqrt{\frac{\mu_{\mathrm{r} 2}}{\varepsilon_{r 2}}}, \\
k_{m 2}=\omega \sqrt{\mu_{0} \varepsilon_{0}} \sqrt{\mu_{r 2} \varepsilon_{r 2}},
\end{gathered}
$$

where $\eta_{0}=377 \Omega$ is the impedance of free space. The input impedance of the short-circuited layer 1 can be expressed as

$$
Z_{\text {in-1 }}=j Z_{m 1} \tan \left(k_{m 1} t_{1}\right) \text {, }
$$

and we can obtain the input impedance of the entire absorption channel as

$$
Z_{\text {in }}=\mathrm{Z}_{m 2} \frac{Z_{i n-1}+j Z_{m 2} \tan \left(k_{m 2} t_{2}\right)}{Z_{m 2}+j Z_{i n-1} \tan \left(k_{m 2} t_{2}\right)} .
$$

Hence, the reflection coefficient can be calculated as

$$
\Gamma=\frac{Z_{0 f s r}-Z_{i n}}{Z_{0 f s r}+Z_{i n}} .
$$

From the ECM analysis, the reflection coefficient is related to the height ratio $h_{1} / h_{2}$ of the magnetic material and the PEC block. Fig. 3(c) depicts the simulated reflection coefficients of the absorption channel in Fig. 3(a) with different height $h_{2}$ of the PEC block when the height $h_{1}$ of the magnetic material is fixed at $8.5 \mathrm{~mm}$. It can be seen that by increasing the height $h_{2}$ from 0 to $5 \mathrm{~mm}$, the reflection peak at $5.45 \mathrm{GHz}$ increases from $-5.5 \mathrm{~dB}$ to $-3.3 \mathrm{~dB}$, due to contribution of the reflective PEC block. The reflections in both the lower and upper absorption bands also increase with the height of PEC block. Nonetheless, wide absorptions with $S_{11}<-10 \mathrm{~dB}$ still maintain in both bands.

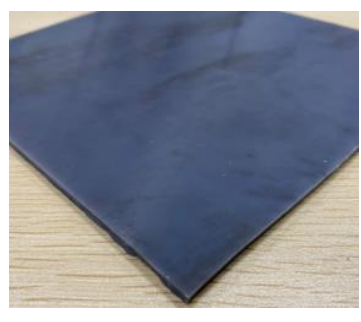

(a)

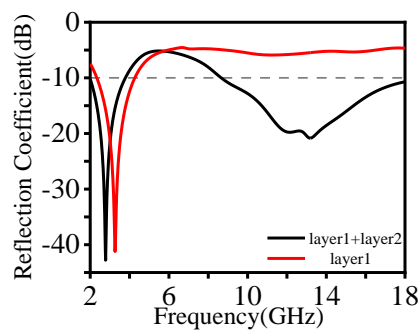

(c)

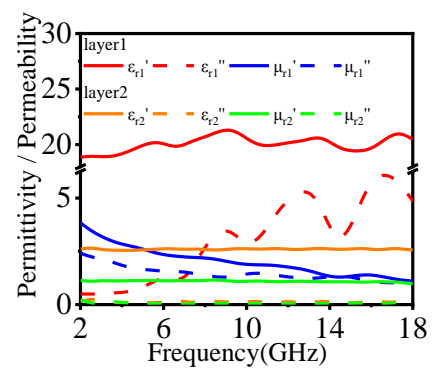

(b)

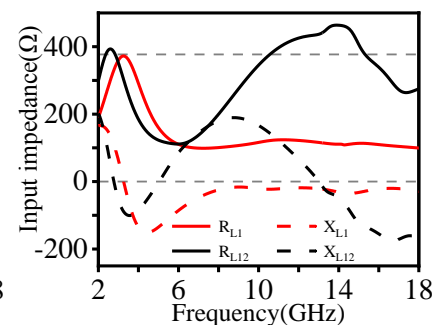

(d)
Fig. 2. Magnetic materials used in our design. (a) Photography of the materials, and (b) permittivity $\left(\varepsilon_{\mathrm{r}}\right)$ and permeability $\left(\mu_{\mathrm{r}}\right)$ of the layer 1 and layer 2 , respectively. (c) Reflection coefficients and (d) input impedances of the grounded magnetic absorbers with layer 2 and without layer 2 . The thickness of layer 1 and layer 2 are $t_{1}=2.6 \mathrm{~mm}$, and $t_{2}=3.7 \mathrm{~mm}$, respectively.

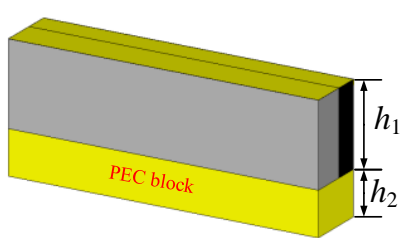

(a)

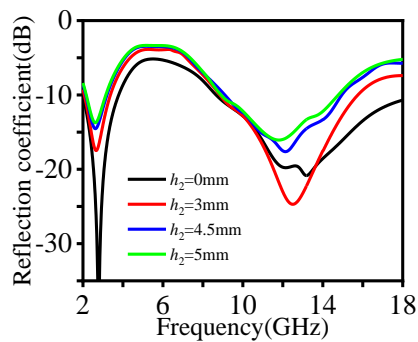

(c)

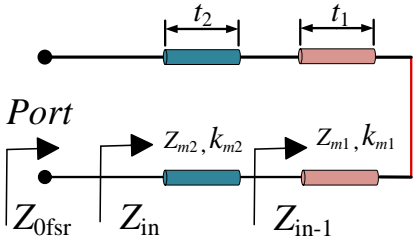

(b)

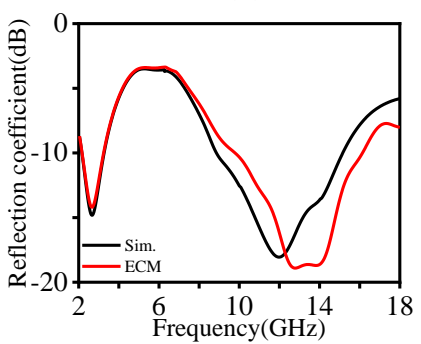

(d)
Fig. 3. (a) Unit-cell geometry of the absorption channel with PEC block, and (b) its equivalent circuit model. (c) Reflection coefficients for different height $h_{2}$ of the PEC block when the height $h_{1}$ of magnetic material is $8.5 \mathrm{~mm}$. (d) Simulated and calculated reflection coefficients $\left(h_{2}=4.5 \mathrm{~mm}\right)$.

The reflection of the absorption path with $h_{2}=4.5 \mathrm{~mm}$ is calculated according to the ECM, as shown in Fig. 3(d), which agrees well with the simulated results, verifying the proposed circuit model. Note that our proposed absorption path design has intrinsic frequency-selective reflection property without introducing any band-stop FSS, enabling lumped-components-free and lithography-free features, which is advantageous for reducing the fabrication complexity and cost of the FSR.

\section{Transmission Path Based on ENZ Tunneling Channels}

The traditional 3-D FSRs usually use PPWs with central metallic via as the transmission part, which require a thickness of half the guided wavelength of transmission frequency. To address this issue on thickness, we introduce the ENZ 
waveguide with tunneling effect as the transmission path in the FSR design (Fig. 1). First, let us discuss the unique transmission property of the waveguide in Fig. 4(a). In a rectangular metallic waveguide, the fundamental transverse-electric $\mathrm{TE}_{10}$ mode is supported with propagation constant

$$
\beta=\sqrt{\left(\frac{2 \pi n f}{c}\right)^{2}-\left(\frac{\pi}{w}\right)^{2}}
$$

where $n$ is the relative refractive index of the dielectric filling the waveguide, $c$ is the speed of light in vacuum, $f$ is the frequency and $w$ is the waveguide width. An effective relative permittivity for the $\mathrm{TE}_{10}$ guided mode in a rectangular waveguide can be defined as [26]

$$
\frac{\varepsilon_{\mathrm{eff}}}{\varepsilon_{0}}=n^{2}-\frac{c^{2}}{4 f^{2} w^{2}} .
$$

As shown in Fig. 4(a), the effective permittivity is frequency dispersive and has a plasma-like property. When the filling dielectric is air $(n=1)$, the wavelength point with zero effective permittivity is calculated as $\lambda_{\mathrm{c}}=2 w$, i. e. $f=4.69 \mathrm{GHz}$.

The height $h_{2}$ of the waveguide should be much less than the height $h_{1}$ of the metal block, ensuring that the EM wave is compressed into the tunnel. Figure 4(b) is the simulated transmissions of the ENZ waveguides with the height $h_{2}$ of 0.3 $\mathrm{mm}$ and $4.5 \mathrm{~mm}$, respectively. When the height $h_{2}$ is $0.3 \mathrm{~mm}$, the transmission peak is located at $4.7 \mathrm{GHz}$, which is near the ENZ frequency. When the height increases to $4.5 \mathrm{~mm}$, the perfect transmission frequency has a blueshift to $5.3 \mathrm{GHz}$, with an effective permittivity of 0.22 [Fig. 4(b)]. This frequency shift phenomenon can be explained by the equivalent circuit model shown in Fig. 4(c). A parallel capacitance $C$ is introduced to model the $E$-step discontinuity between the free space and the channel, while a parallel inductance $L$ is used to model the $H$-step discontinuity [25]. The channel is modeled as a transmission line section with propagation constant in Eq. (5), length of $t$ and characteristic impedance of

$$
Z_{w}=\frac{\eta_{0}}{\sqrt{\varepsilon_{e f f}}} \frac{h_{2}}{w} .
$$

At the resonance frequency where the total impedance of $L-C$ parallel circuit is infinite, and when the characteristic impedance $Z_{\mathrm{w}}$ is matched to the port impedance $Z_{0 \mathrm{fs} r}$, perfect transmission can be obtained. Then we have $\varepsilon_{\text {eff }}=$ $1 /\left(h_{1} / h_{2}+1\right)^{2}$ for perfect transmission, which increases as the height $h_{2}$ increases, resulting in the blue-shift of the transmission frequency. Thus, when the height $h_{2}$ of the channel is much less than the height $h_{1}$ of the PEC block $\left(h_{1} / h_{2}\right.$ goes to infinity), the frequency with $\varepsilon_{\text {eff }} \approx 0$ corresponds to tunneling frequency. It can be seen in Fig. 4(b) that the calculated results fit well with the simulated ones in spite of small discrepancy in the high band, which is caused by the high order modes not considered in the ECM. The capacitance and inductance are $C=0.9 \mathrm{pF}$ and $L=1.16 \mathrm{nH}$ for $h_{2}=0.3 \mathrm{~mm}$, and $C=$ $0.14 \mathrm{pF}, L=5.7 \mathrm{nH}$ for $h_{2}=4.5 \mathrm{~mm}$ case, which are obtained based on the curve-fitting of the calculated transmissions with the full-wave simulation results. The transmission curve is broadened when the height increases [see Fig. 4(b)], since the bandwidth of the transmission is proportional to $L / C$. To verify the tunneling effect at the ENZ frequency further, simulation plot of distributions of the electric field are given in Fig. 4(d). It shows that little amplitude change (left panel) and zero phase delay (right panel) occur within the narrow channel with a

length of $0.1 \lambda_{\mathrm{c}}$ at the ENZ frequency $4.69 \mathrm{GHz}$, consistent with propagation in an ENZ metamaterial [28]-[31]. The electric field is squeezing into the narrow channel with enhanced field amplitude and zero phase delay, which is crucial for realizing frequency-selective perfect transmission in arbitrarily thin thickness.

In Fig. 5 (a), the influence of the length of waveguide (thickness $t$ ) on the scattering coefficients is given. When the thickness increases from $0.3 \mathrm{~mm}$ to $6.3 \mathrm{~mm}$, the transmission peak (reflection dip) moves from 5.58 to $5.3 \mathrm{GHz}$, with only $5 \%$ of variation. This stability of transmission frequency on the length is attributed to the near-zero phase delay in the ENZ channel as depicted in Fig. 4(d). According to Eq. (6), the ENZ frequency is related to the waveguide width $w$. Thus, the transmission frequency increases as the width decreases, as verified in Fig. 5(b).

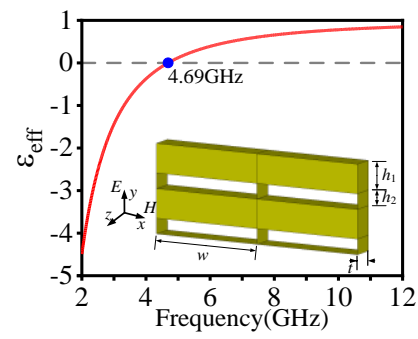

(a)

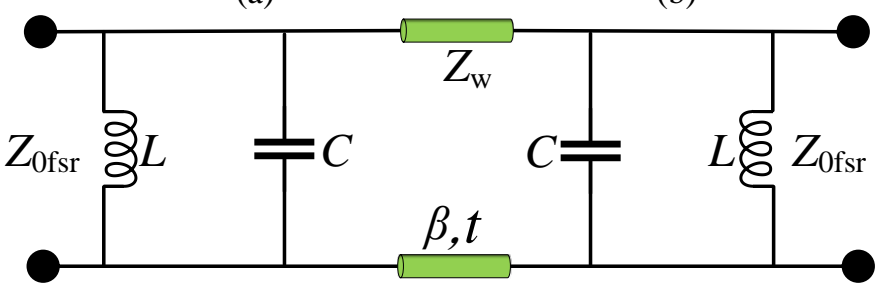

(c)

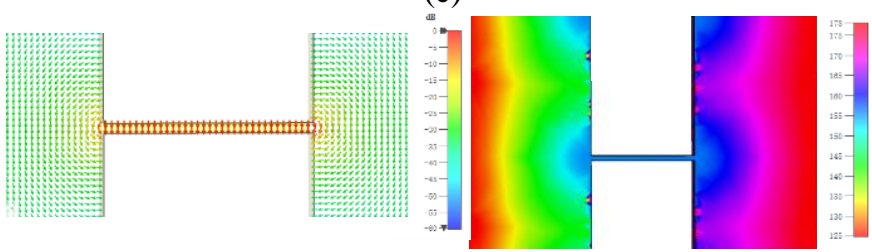

(d)

Fig. 4. (a) The frequency dispersive effective permittivity of the waveguide ( $2 \times 2$ array in the inset). (b) The simulated and calculated (circuit model) transmission coefficients of the ENZ metallic waveguide with different height $h_{2}$. (c) The equivalent circuit model of the frequency-selective transmission structure. (d) The electric field vector (left panel) and phase (right panel) distributions at the tunneling frequency $4.69 \mathrm{GHz}\left(h_{2}=0.3 \mathrm{~mm}\right)$.

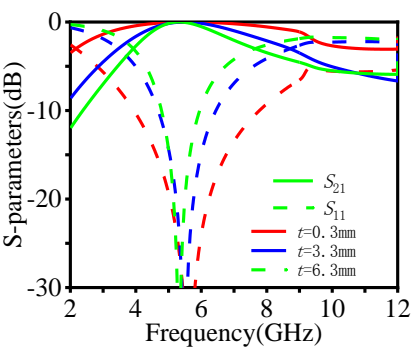

(a)

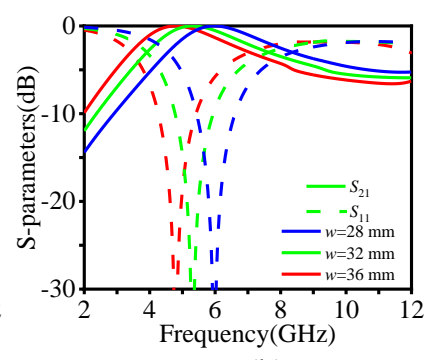

(b)
Fig. 5. The simulated transmission and reflection coefficients of the ENZ metallic waveguide with (a) different length $t$, and (b) different width $w$. 


\section{Integrated Design}

By integrating the absorption and transmission channels described above, a 3-D FSR is constructed [the inset of Fig. 6(a)]. In our absorption path, the AFSR structure has an intrinsic reflection peak at $5.45 \mathrm{GHz}$. Hence, we need to design the transmission window near this fixed frequency to reduce the insertion loss. Then the transmission waveguide width is set as $27.5 \mathrm{~mm}$ initially, which is half the transmission wavelength. To suppress the reflection of the integrated FSR at the neighboring frequencies of the reflection band of the AFSR structure, the bandwidth of transmission path should be designed to match with the frequency-selective reflection bandwidth of the absorption path. Therefore, the height of the ENZ waveguide is set as $h_{2}=4.5 \mathrm{~mm}$ initially. The thickness $t$ of the transmission path is selected as $6.3 \mathrm{~mm}$, which is the same as the absorption path, reducing the overall thickness to the utmost extent. The whole structure is optimized in full-wave simulations to obtain low insertion loss and wideband absorptions at both sides of the transmission band. The optimized geometrical parameters are $h_{1}=8.5 \mathrm{~mm}, h_{2}=4.5 \mathrm{~mm}$ and $w=32 \mathrm{~mm}$. The ECM of the integrated 3-D FSR is established in Fig. 6(a), by connecting the two subnetworks of the absorption and transmission paths in series.

The $S$-parameters of the FSR for the TE polarization under the normal incidence are simulated and analytically examined, as shown in Fig. 6(b). The 3-D FSR exhibits a transmission window at $5.24 \mathrm{GHz}$ with an insertion loss of $1 \mathrm{~dB}$ and the transmission band with $\left|S_{21}\right| \geq-3 \mathrm{~dB}$ ranges from 4.2 to 6.52 $\mathrm{GHz}$ (fractional bandwidth (FBW) of 43.4\%). The absorption band covers from 2.3 to $12 \mathrm{GHz}(135.6 \% \mathrm{FBW})$, in spite of two reflection peaks at $4.2 \mathrm{GHz}\left(\left|S_{11}\right|=-8.5 \mathrm{~dB}\right)$ and $7.1 \mathrm{GHz}\left(\left|S_{11}\right|=\right.$ $-9.1 \mathrm{~dB})$. Since the waveguide has an additional transmission band near $16.8 \mathrm{GHz}$ caused by conventional Fabry-Perot resonances [26], [27], for the sake of concentrating on the ENZ transmission effect, the $S$-parameters from 12 to $18 \mathrm{GHz}$ of the integrated FSR is not discussed throughout this work. As shown in Fig. 6(b), the analytical results match well with the simulated ones in the interested band.

The $S$-parameters of the FSR under the oblique incidences are also examined and given in Fig. 6(c). In the lower band below the transmission frequency, the simulated results are stable for both $15^{\circ}$ and $30^{\circ}$ incidence, with a transmission window (insertion loss $0.9 \mathrm{~dB}$ ) at $5.2 \mathrm{GHz}$. However, when the incidence angle increases, the reflection in the upper band (above $5.2 \mathrm{GHz}$ ) increases, even larger than $-7 \mathrm{~dB}$ for $30^{\circ}$ scenario. Moreover, an additional transmission peak appears obviously at $10 \mathrm{GHz}$ for $30^{\circ}$ incidence $\left(\left|S_{21}\right|=-6.1 \mathrm{~dB}\right)$, originating from the Fabry-Perot resonance transmission mode of the ENZ waveguide structure, which shifts towards lower frequency for a larger incidence angle.

It should be mentioned that according to the Floquet theory, infinite numbers of harmonics are scattered by periodic structures, including propagating modes and evanescent modes [32]. The propagating modes are scattered into far-field, and the evanescent modes are absorbed by the lossy structure ultimately. Therefore, only the propagating modes contribute to the reflection and transmission coefficients. The tangential wave number of the reflected harmonics can be calculated as $k_{\mathrm{rx}}=k_{0} \sin \theta+2 \pi \mathrm{m} / D$, where $\mathrm{m}$ is the harmonic order, and $D$ is the

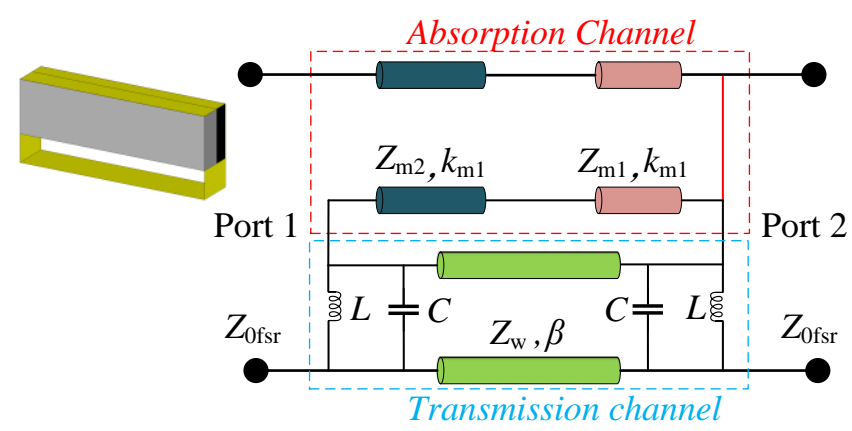

(a)

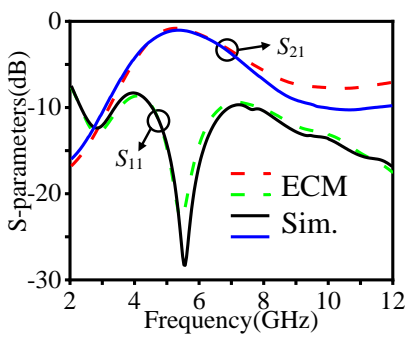

(b)

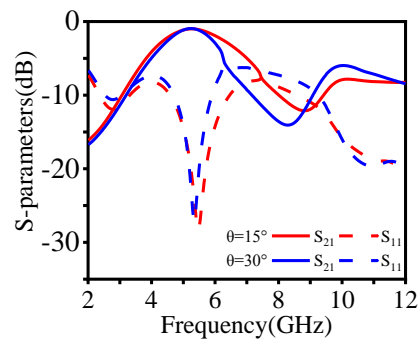

(c)
Fig. 6. (a) ECM of the proposed FSR. (b) Simulated and calculated (circuit model) $S$-parameters under the normal incidence. (c) Simulated $S$-parameters under the oblique incidences.

period of unit cell. When the tangential wave number satisfies the condition of $\left|k_{\mathrm{rx}}\right|>k_{0}$, the higher-order harmonics are evanescent, otherwise, they are propagating. In this paper, the period $D$ is $32 \mathrm{~mm}$, more than one wavelength in the band above $9.375 \mathrm{GHz}$. For normal incidence, the $\mathrm{m}= \pm 1$ order diffraction modes are evanescent below $9.375 \mathrm{GHz}$ and propagating between 9.375 and $12 \mathrm{GHz}$. The transmission and reflection components in the $m= \pm 1$ orders are less than $-17 \mathrm{~dB}$ in the simulation (not shown here), which means that the high-order scattered propagating harmonics are negligible in the FSR. For oblique incidence up to $30^{\circ}$, only the $\mathrm{m}=0$ and $\mathrm{m}=-1$ orders are propagating in the band between 9 and $12 \mathrm{GHz}$. In the simulation, the transmission and reflection components in the $m=-1$ order diffracted modes are less than $-8 \mathrm{~dB}$ and $-12 \mathrm{~dB}$, respectively (not shown here). Consequently, the high-order diffraction modes are low-level in the interested band, unable to make a decisive difference to the zero-order transmission and reflection performance of the FSR.

\section{MEASUREMENTS AND DISCUSSIONS}

To verify the performance of the proposed ultrathin FSR, the structure is fabricated and measured, as shown in Fig. 7. The ferrite absorber has a size of $200 \mathrm{~mm} \times 200 \mathrm{~mm}$ and a thickness of $6.3 \mathrm{~mm}$. First, we use a customized cutting die to cut away 15 rectangular strips with the size of $192 \mathrm{~mm} \times 4.5 \mathrm{~mm}$ from the complete absorbing material. The period of the slot array is $13 \mathrm{~mm}$. Then copper foils are pasted on the back of the magnetic layer 1 and the inner walls of the rectangular hollow waveguides. Hard strips made of copper-coated PVC with a size of $6.3 \mathrm{~mm} \times 4.5 \mathrm{~mm}$ are inserted into each $192 \mathrm{~mm}$-wide rectangular slot to divide it into six $32 \mathrm{~mm}$-wide rectangular waveguides. Finally, a prototype of the FSR with a $15 \times 6$ array is fabricated.

As shown in Fig. 7(b) and 7(c), a free-space method is used 


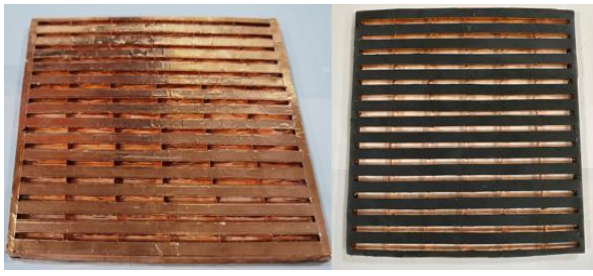

(a)

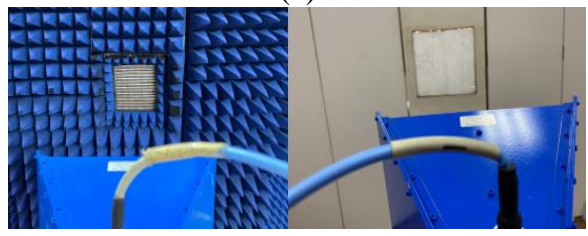

(b)

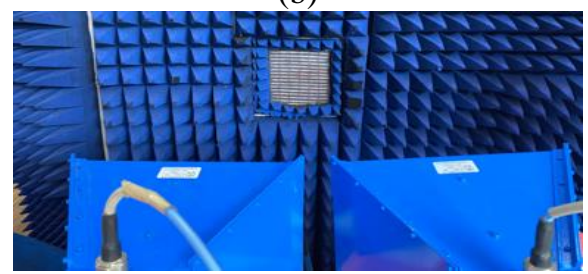

(c)

Fig. 7. Photographs of (a) the fabricated FSR, (b) the transmission and (c) reflection test setups.

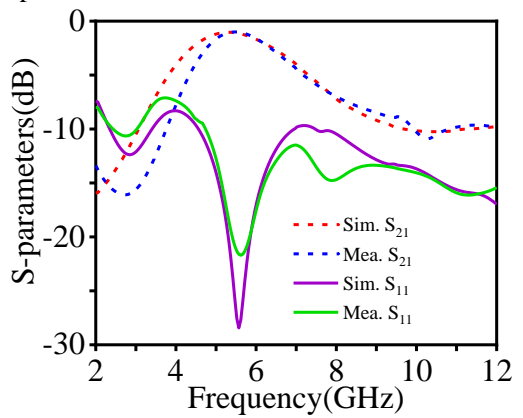

(a)

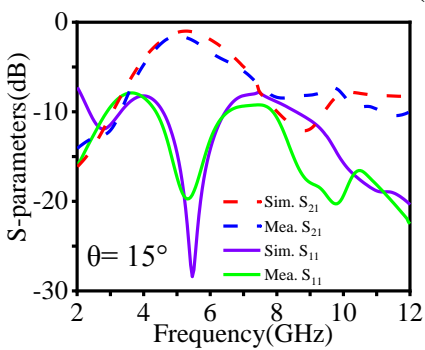

(b)

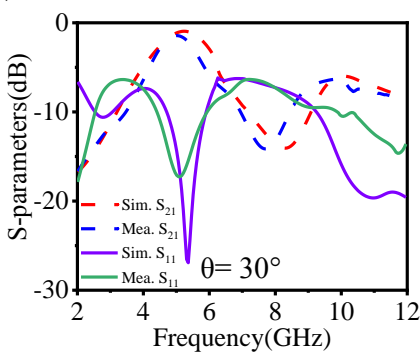

(c)
Fig. 8. Measured and simulated results of the fabricated FSR (a) under the normal incidence $\left(8^{\circ}\right.$ incidence angle is adopted approximately in the reflection test), (b) $15^{\circ}$ and (c) $30^{\circ}$ incidence. The electric filed is fixed in the $y$-direction.

to measure the reflection $\left(S_{11}\right)$ and transmission $\left(S_{21}\right)$ coefficients, which is similar to the one used in [5], [13]. In the transmission test, two horn antennas are placed on the two sides of the absorbing wall with a same distance of 2 meters [Fig. 7(b)]. The sample is put at the center of the window of absorbing wall. Additionally, for measurements for oblique incidence, the absorbing wall with the sample is rotated. The results are normalized with the transmissions from the absorbing wall without the sample. In the measurement of reflection, as shown in Fig. 7(c), the antennas are located at the same side of the absorbing screen. A metallic plate that has a size identical to the prototype is tested for normalization. A time gate is employed to smooth the results by filtering the unwanted scattering noises.

In Fig. 8(a), it is observed that the measured results have good agreement with the simulated ones, with a passband at 5.2 GHz. The insertion loss is $1.02 \mathrm{~dB}$, and the transmission band with $\left|S_{21}\right| \geq-3 \mathrm{~dB}$ ranges from 4.6 to $6.6 \mathrm{GHz}$ (FBW of $35.7 \%$ ). Moreover, the measured reflection is below $-10 \mathrm{~dB}$ from 2.3 $\mathrm{GHz}$ to $12 \mathrm{GHz}$ (FBW of $135.6 \%$ ), with the exception of the reflection peak $(-7 \mathrm{~dB})$ at $4 \mathrm{GHz}$, which is also observed in the simulation. The small discrepancy between the measured results and the simulated ones should be ascribed to the imperfect fabrication of the sample and the test errors.

The angular stability of the FSR is also examined in Figs. 8(b) and 8(c). Similar with the simulation results, at oblique incident angles, an additional transmission peak appears in the upper band and its transmissivity increases for a larger incidence angle. Moreover, the reflection also rises in the upper band when the incidence angle increases up to $30^{\circ}$. Nonetheless, absorptions more than $80 \%\left(\left|S_{11}\right|<-7 \mathrm{~dB}\right)$ and a transmissivity of $79 \%\left(\left|S_{21}\right|=-1.05 \mathrm{~dB}\right)$ at the transmission frequency are maintained from $2.3 \mathrm{GHz}$ to $12 \mathrm{GHz}$ for both oblique angles. In general, the FSR shows acceptable angular stability below $30^{\circ}$ incidence angle in the whole band interested.

\section{EXTREMELY THIN, DUAL-POLARIZED AND DUAL-BAND RASORBER DESIGNS INSPIRED BY ENZ TUNNELING CONCEPT}

\section{A. Extremely Thin Design}

In [21], a record thin 3-D FSR working from $10 \mathrm{MHz}$ to 1 $\mathrm{GHz}$ whose thickness is $0.086 \lambda_{\mathrm{c}}(47 \mathrm{~mm})$, where $\lambda_{\mathrm{c}}$ is the free-space wavelength at the center frequency $(0.548 \mathrm{GHz})$ of the passband, has been proposed. A SN-20 ferrite material [33] is used to implement the absorption channel, which is able to absorb EM waves ranging from $10 \mathrm{MHz}$ to $1 \mathrm{GHz}$, with a thickness of $6.7 \mathrm{~mm}$. To reduce the insertion loss, a circuit resonator constructed by lumped inductor and capacitor in series is employed, $4 \mathrm{~mm}$ away from the absorbing materials. Although the thickness of the absorption path is $11.2 \mathrm{~mm}$, the transmission channel constructed by slow wave structure in [21] is still $47 \mathrm{~mm}$-long in the propagation direction, hindering further decrease of the overall thickness.

Benefiting from tunneling effect independent of propagation length in ENZ waveguides, an extremely thin design is realized. By using the same design of absorption path as that in [21], replacing the parallel plate waveguide and additional slow wave structure in [21] with a rectangular ENZ waveguide with

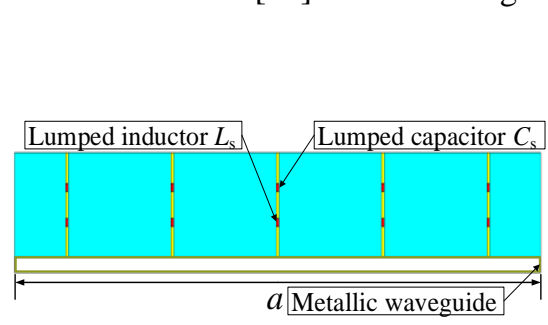

(a)

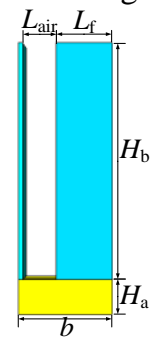

(b)
Fig. 9. Structure of the extremely thin FSR. (a) Front view and (b) side view of a unit cell. (Physical dimensions: $a=300 \mathrm{~mm}, b=11.2 \mathrm{~mm}, L_{\mathrm{f}}=6.7 \mathrm{~mm}, L_{\mathrm{air}}=4 \mathrm{~mm}$, $H_{\mathrm{a}}=4.2 \mathrm{~mm}, H_{\mathrm{b}}=28.8 \mathrm{~mm}, L_{\mathrm{s}}=105 \mathrm{nH}, C_{\mathrm{s}}=0.3 \mathrm{pF}$, the width of microstrip lines $w_{\mathrm{s}}=0.5 \mathrm{~mm}$. The microstrip lines are printed on a $0.5-\mathrm{mm}$-thick substrate with permittivity of $\varepsilon_{\mathrm{r}}=3.55, t_{\mathrm{s}}=0.5 \mathrm{~mm}$ ) 


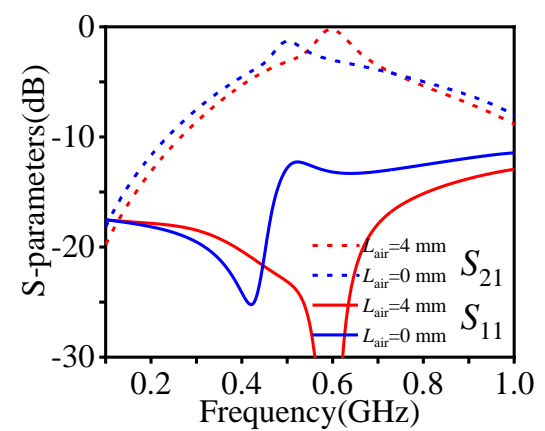

Fig. 10. The simulated $S$-parameters of the designs with different $L_{\text {air }}$. The length $b$ of the waveguide is also varying with $L_{\mathrm{air}}\left(b=L_{\mathrm{f}}+L_{\mathrm{air}}+t_{\mathrm{s}}, L_{\mathrm{f}}=6.7 \mathrm{~mm}\right.$, $\left.t_{\mathrm{s}}=0.5 \mathrm{~mm}\right)$

a length equal to the absorption path length, as shown in Fig. 9, the thickness of the FSR can shrink dramatically into $11.2 \mathrm{~mm}$, averting the increase of thickness caused by the transmission path.

The simulation results of the extremely thin design in Fig. 9 are shown in Fig. 10. The transmission band $\left(\left|S_{21}\right| \geq-3 \mathrm{~dB}\right)$ ranges from 0.51 to $0.68 \mathrm{GHz}$ (FBW 28.6\%) with a minimum insertion loss of $0.23 \mathrm{~dB}$ at $0.596 \mathrm{GHz}$, better than the performances (FBW 27\%, insertion loss $0.27 \mathrm{~dB}$ ) in [21]. Furthermore, the reflection is lower than $-13 \mathrm{~dB}$, ranging from the whole interested band $(10 \mathrm{MHz}$ to $1 \mathrm{GHz})$, similar with that in [21]. Strikingly, the thickness of our design is only $0.022 \lambda_{\mathrm{c}}$, showing significant advantage in thickness compared with the design $\left(0.086 \lambda_{c}\right)$ in [21]. Interestingly, the thickness can be reduced further into $7.2 \mathrm{~mm}$ by eliminating the space between the bandstop FSS layer and the ferrite, i.e. $L_{\text {air }}=0 \mathrm{~mm}$. As shown in Fig. 10, the low reflection with $\left|S_{11}\right| \leq-11.4 \mathrm{~dB}$ is maintained in the whole band, and the transmission band moves to $0.456-0.6 \mathrm{GHz}$ (FBW 27.3\%) with a transmission peak at $0.502 \mathrm{GHz}$ (insertion loss of $1.3 \mathrm{~dB}$ ). The FSR has a record ultrathin thickness of $0.012 \lambda_{\mathrm{c}}$ with a comparable low insertion loss, to the best of our knowledge.

\section{B. Dual-Polarized Design}

The single-polarized FSR in Section II is extended further into a dual-polarized design. The unit-cell structure is shown in Fig. 11(a), in which two identical rectangular metallic waveguides are introduced and arranged in rotationally symmetry in a unit cell, ensuring the polarization-insensitive property of the design. The waveguide has a width of $30 \mathrm{~mm}$ and a height of $0.5 \mathrm{~mm}$. The period of the dual-polarized design is $30.5 \mathrm{~mm}$. The absorption path is composed of two layers, including the top layer with thickness of $4 \mathrm{~mm}$ and permittivity of 4.4, and the metal-grounded ferrite layer 1 with thickness of $3 \mathrm{~mm}$. The overall thickness of the FSR is $t=7 \mathrm{~mm}$.

The simulated $S_{11}$ and $S_{21}$ results of the FSR under normal incidence for different polarization angles are shown in Fig. 11(b). The E-field direction is scanned in the $x-y$ plane. Due to the rotationally symmetry in the $x-y$ plane, the frequency responses are polarization-stable, as shown in Fig. 11(b). A transmission peak at $4.93 \mathrm{GHz}$ with insertion loss of $1.58 \mathrm{~dB}$ is obtained between two wide absorption bands. The passband with $\left|S_{21}\right| \geq-3 \mathrm{~dB}$ is from 4.88 to $4.98 \mathrm{GHz}$, with FBW of $2 \%$. The high selectivity should be due to the small height of waveguide $(h=0.5 \mathrm{~mm})$. The reflection is lower than $-10 \mathrm{~dB}$ in the bands of $2.78-3.42 \mathrm{GHz}$ and $8.15-12 \mathrm{GHz}$. The reflection
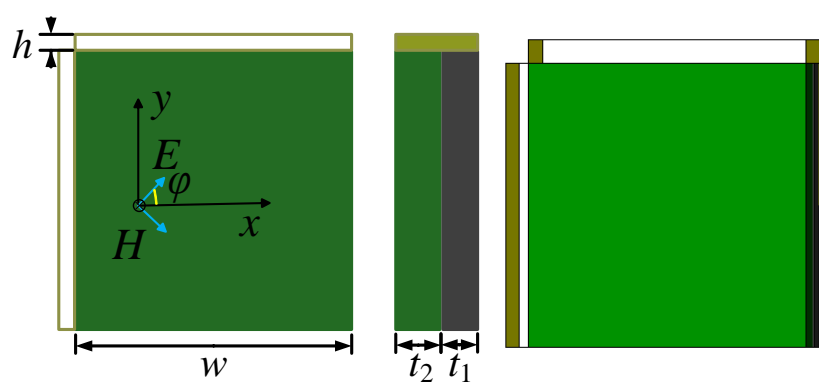

(a)

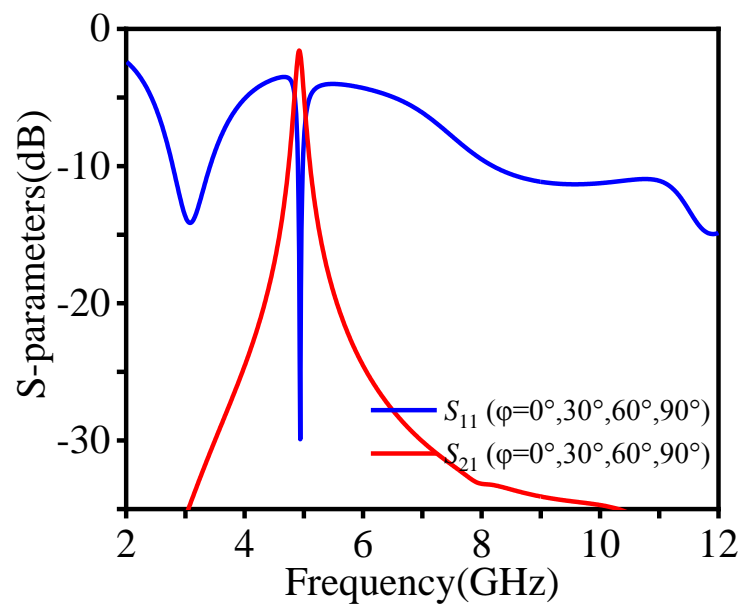

(b)

Fig. 11. (a) The dual-polarized structure. (Physical dimensions: $w=30 \mathrm{~mm}$, $h=0.5 \mathrm{~mm}, t_{1}=3 \mathrm{~mm}, t_{2}=4 \mathrm{~mm}$.) (b)The simulated $S$-parameters under TE incidences with different polarization angles $\varphi$.

peaks near the transmission band is higher than the single-polarized one, which is caused by the increase of area ratio of the AFSR structure in a unit cell.

\section{Dual-Band Design}

The FSRs with two transmission bands, i.e. dual-band FSRs, are desirable for various scenarios. Most of the previous dual-band FSRs [10], [12] are 2-D design, where 2-D FSSs consisting of multiple series/parallel $L C$ resonators with two impedance poles are implemented to obtain dual-band transmission. Therefore, the 2-D design is usually complicated and the transmission bands are interacted with each other strongly, limiting the design flexibility. In the 3-D FSR concept, the absorption and transmission paths can be designed independently, facilitating the flexibility for different FSR designs. However, the 3-D dual-band FSR is still challenging, since the conventional PPW transmission path is not suitable for dual-band transmission and new transmission structures are required [34].

Since the ENZ waveguide has a transmission peak at the cutoff frequency which is related to its width, it should be easy to realize dual-band transmission by utilizing two ENZ waveguides with different widths in single structure. As depicted in Fig. 12(a), two metallic waveguides with widths $w_{1}=35 \mathrm{~mm}$ and $w_{2}=28 \mathrm{~mm}$, and heights of $h_{2}=2.25 \mathrm{~mm}$ and $h_{3}=2.25 \mathrm{~mm}$ are arranged up and down. Two metallic blocks are placed on both sides of the narrower waveguides. Then the double-waveguide combination is integrated with the same absorption structure in Section II. From the simulation results 
in Fig. 12(b), two transmission peaks at 4.42 and $5.79 \mathrm{GHz}$ with insertion loss of 1.22 and $1.15 \mathrm{~dB}$, respectively, are observed. There are two bands with reflection lower than $-10 \mathrm{~dB}$ on both sides of the transmission bands, corresponding to two high-absorption bands. In addition, a reflection peak between the two transmission bands with $\left|S_{11}\right|=-4 \mathrm{~dB}$ appears, since the two transmission windows are located in the single frequency-selective reflection band of the ferrite-based absorption path. It is believed that by designing multi-band AFSR structures [3], [35] and ENZ waveguide combination with multi-band transmissions overlapping with the multiple reflection bands of the AFSR structures, the in-between reflection peak should be suppressed ultimately. Besides, the insertion loss can also be reduced further by increasing the reflectivity at the reflection bands of the AFSR structure to bypass more losses. In short, owing to the unique properties of the ENZ tunneling channel, including weak dependence over its geometry and weak interference between different ENZ tunneling channels due to their enclosed constructions [26], the ferrite-ENZ integrated scheme is powerful for designing dual-band and even multi-band FSRs in a simple and flexible way.

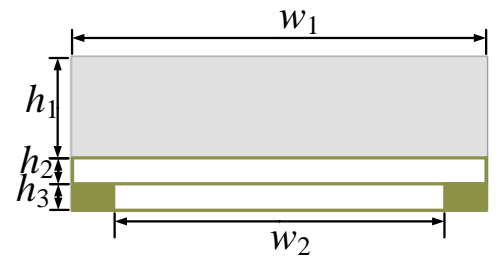

(a)

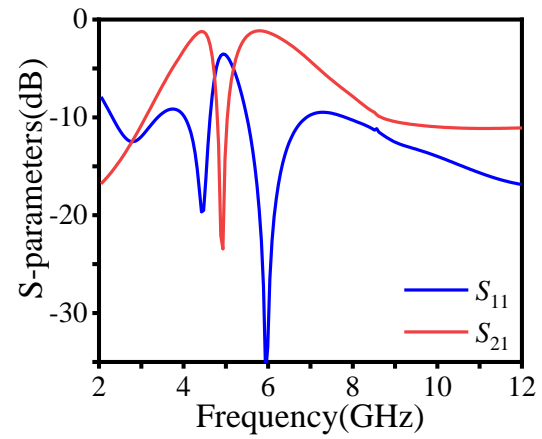

(b)

Fig. 12. (a) The front view of the unit cell. (Physical dimensions: $h_{1}=8.5 \mathrm{~mm}, h_{2}$ $=h_{3}=2.25 \mathrm{~mm}, w_{1}=35 \mathrm{~mm}, w_{2}=28 \mathrm{~mm}$ ) (b) The simulated $S$-parameters of the design. Yellow denotes metal, grey denotes dielectric layer.

TABLE I

COMPARISONS BETWEEN OUR FSRS AND OTHER REPORTED FSRS

\begin{tabular}{|c|c|c|c|c|c|c|}
\hline \multicolumn{2}{|c|}{ Ref. } & $\begin{array}{l}\text { Insertion } \\
\operatorname{loss}(\mathrm{dB})\end{array}$ & $\begin{array}{c}\text { Thickness } \\
\left(\lambda_{c}\right)\end{array}$ & Pol. & $\begin{array}{c}\text { Lumped } \\
\text { component- } \\
\text { free } \\
\end{array}$ & $\begin{array}{l}\text { Lithography- } \\
\text { free }\end{array}$ \\
\hline \multicolumn{2}{|c|}{ [3] } & 0.26 & 0.299 & Single & No & No \\
\hline \multicolumn{2}{|c|}{ [19] } & 0.8 & 0.303 & Single & Yes & No \\
\hline \multicolumn{2}{|c|}{ [20] } & 1.2 & 0.242 & Single & Yes & No \\
\hline \multicolumn{2}{|c|}{ [21] } & 0.27 & 0.086 & Single & No & No \\
\hline \multicolumn{2}{|c|}{ [23] } & 1.26 & 0.312 & Dual & Yes & No \\
\hline \multirow{3}{*}{ Ours } & 1\# & 1.05 & 0.108 & Single & Yes & Yes \\
\hline & 2\# & 0.23 & 0.022 & Single & No & No \\
\hline & 3\# & 1.58 & 0.115 & Dual & Yes & Yes \\
\hline
\end{tabular}

\footnotetext{
${ }^{1} \lambda_{\mathrm{c}}$ is the free-space wavelength at the center frequency of the passband.

${ }^{2} 1$ \# represents the original design, $2 \#$ represents the extremely thin design, $3 \#$ represents the symmetrical dual-polarized design.
}

Finally, in order to show the advantages and characteristics of the ENZ-based 3-D FSRs in our work more intuitively, the parametric comparison of the designs with previous reported FSRs is listed in Table I. Obviously, compared with other structures, our designs feature ultrathin thickness, because the overall thickness can be designed depending on the thickness of the absorption path, which can be ultrathin when magnetic absorbers are applied. For instance, the 2\# design has a record thin thickness of $0.022 \lambda_{c}$ by integrating the $\mathrm{SN}-20$ ferrite absorber with ENZ transmission waveguides. Besides, the ENZ-based rasorber can be easily extended into dual-band and dual-polarized designs, showing remarkable flexibility. Moreover, in the 1\# and 3\# designs, no lumped components and no lithographic patterns are involved, greatly facilitating the fabrication and reducing the cost, which has a broad prospect in the future practical applications.

\section{CONCLUSION}

By applying the thin wideband ferrite material in the absorption channel and implanting ENZ tunneling waveguides as the transmission channel, an ultrathin 3-D FSR with a transmission window located within the wide absorption band are proposed, fabricated and measured. With a dielectric layer on top of the ferrite material, the absorption path exhibits intrinsic frequency-selective reflection peak within a wide absorption band, avoiding using any band-stop FSS as bypassing network. In addition, the ENZ tunneling waveguide has a transmission peak at the cutoff frequency of $\mathrm{TE}_{10}$ mode, independent on the length of waveguide due to the near-zero phase delay at the ENZ frequency. ECMs are established to demonstrate the operating principle of the ferrite-based AFSR structure and the ENZ waveguides, formulating a guideline for designing the physical parameters. These properties simplify the integrated design dramatically and bring great benefits in term of cost in the lithography-free and lumped-components-free designs. Furthermore, to demonstrate the great superiority and flexibility of the ferrite-ENZ-integrated design strategy, several FSR designs with extremely thin thickness, dual-polarized characteristic and dual transmission bands are presented, respectively. A record thin rasorber with a thickness of $0.022 \lambda_{\mathrm{c}}$ is realized in a simple form, showing remarkable advantages in thickness over the traditional 2-D and 3-D rasorbers. The original design can be extended to dual-polarized and dual-band designs easily, because the waves with different frequencies or polarizations can transmit through corresponding waveguides separately, without interfering each other due to the closed metallic structure. It is believed that the performance can be improved further by employing other ferrites and band-stop FSSs in the absorption path, which is worthy of investigation in the future.

\section{REFERENCES}

[1] B. A. Munk, Frequency Selective Surfaces: Theory and Design. New York, NY, USA: Wiley, 2000

[2] F. Costa and A. Monorchio, "A Frequency Selective Radome With Wideband Absorbing Properties," IEEE Trans. Antennas Propag. vol. 60, no. 6, pp. 2740-2747, Jun. 2012.

[3] A. A. Omar, H. Huang and Z. Shen, "Absorptive Frequency-Selective Reflection/Transmission Structures: A Review and Future Perspectives," IEEE Antennas and Propag. Mag., vol. 62, no. 4, pp. 62-74, Aug. 2020. 
[4] Y. Shang, Z. Shen and S. Xiao, "Frequency-Selective Rasorber Based on Square-Loop and Cross-Dipole Arrays," IEEE Trans. Antennas Propag., vol. 62 , no. 11, pp. 5581-5589, Nov. 2014.

[5] L. Wu, S. Zhong, J. Huang, and T. Liu, "Broadband Frequency-Selective Rasorber with Varactor-Tunable Interabsorption Band Transmission Window," IEEE Trans. Antennas Propag., vol. 67, no. 9, pp. 6039-6050, Sep.2019.

[6] Q. Chen, D. Sang, M. Guo and Y. Fu, "Frequency-selective rasorber with inter-absorption band transparent window and interdigital resonator," IEEE Trans. Antennas. Propag., vol. 66, no. 8, pp. 4105-4114, Aug. 2018.

[7] Q. Guo, Z. Li, J. Su, L. Y. Yang, and J. Song, "Dual-polarization absorptive/transmissive frequency selective surface based on tripole elements," IEEE Antennas Wireless Propag. Lett., vol. 18, no. 5, pp. 961-965, May 2019.

[8] Y. Han, L. Zhu, Y. Chang, and B. Li, "Dual-polarized bandpass and band-notched frequency-selective absorbers under multimode resonance," IEEE Trans. Antennas Propag., vol. 66, no. 12, pp. 7449-7454, Dec. 2018.

[9] S. Zhong, L. Wu, T. Liu, J. Huang, W. Jiang, and Y. Ma, "Transparent transmission-selective radar-infrared bi-stealth structure,”Opt. Express, vol. 26, no.13, pp. 16466-16476, Jun. 2018.

[10] M. Guo, Q. Chen, Z. Sun, D. Sang and Y. Fu, "Design of Dual-Band Frequency-Selective Rasorber," IEEE Antennas and Wireless Propag. Lett., vol. 18, no. 5, pp. 841-845, May 2019.

[11] Q. Chen, D. Sang, M. Guo and Y. Fu, "Miniaturized Frequency-Selective Rasorber With a Wide Transmission Band Using Circular Spiral Resonator," IEEE Trans. Antennas Propag., vol. 67, no. 2, pp. 1045-1052, Feb. 2019.

[12] M. Guo, Q. Chen, D. Sang, Y. Zheng and Y. Fu, "Dual-Polarized Dual-Band Frequency Selective Rasorber With Low Insertion Loss," IEEE Antennas and Wireless Propag. Lett., vol. 19, no. 1, pp. 148-152, Jan. 2020

[13] Z. Xue, S. Zhong and Y. Ma, "Graphene-FSS Hybrid Absorptive Structure with Amplitude/Frequency Dual-Modulated Passband," IEEE Antennas and Wireless Propag. Lett., IEEE Antennas and Wireless Propag. Lett., vol. 20, no. 9, pp. 1711-1715, Sept. 2021.

[14] B. Li and Z. Shen, "Wideband 3D frequency selective rasorber," IEEE Trans. Antennas Propag., vol. 62, no. 12, pp. 6536-6541, Dec. 2014.

[15] Y. Yu, Z. Shen, T. Deng, and G. Luo, "3D frequency selective rasorber with wide upper absorption Band," IEEE Trans. Antennas Propag., vol. 65, no. 8, pp. 4363-4367, Aug. 2017.

[16] Y. Yu et al., "3D Absorptive Frequency-Selective Reflection and Transmission Structures With Dual Absorption Bands," IEEE Access, vol. 6, pp. 72880-72888, 2018.

[17] H. Zhou et al., "Experimental demonstration of an absorptive/transmissive FSS with magnetic material," IEEE Antennas Wireless Propag. Lett., vol. 13, pp. 114-117, 2014.

[18] T. Deng, Y. Yu, Z. Shen, and Z. N. Chen, "Design of 3-D multilayer ferrite-loaded frequency-selective rasorbers with wide absorption bands," IEEE Trans. Microw. Theory Techn., vol. 67, no. 1, pp. 108-117, Jan. 2019.

[19] A. A. Omar, J. Kim and W. Hong, "A 3-D Lumped-Components-Free Absorptive Frequency-Selective Transmission Structure Featuring Very Wide Two-Sided Absorption Bandwidths," IEEE Antennas and Wireless Propag. Lett., vol. 19, no. 5, pp. 761-765, May 2020.

[20] Y. Yu, G. Q. Luo, W. Yu, J. Tong and Z. Shen, "3-D Frequency-Selective Rasorber Based on Magnetic Material and Meander Line," IEEE Trans. Antennas Propag., vol. 68, no. 11, pp. 7694-7699, Nov. 2020.

[21] Y. Wang, S. Qi, Z. Shen and W. Wu, "Ultrathin 3-D Frequency Selective Rasorber With Wide Absorption Bands," IEEE Trans. Antennas Propag., vol. 68, no. 6, pp. 4697-4705, Jun. 2020.

[22] Eccosorb® FGM-125, Laird ${ }^{\mathrm{TM}}$, datasheet. Accessed on: Oct. 1, 2017. [Online].Available:http://www.eccosorb.eu/products/eccosorb/eccosorb -fgm-125.

[23] Y. Wang, M. Wang, Z. Shen and W. Wu, "3-D Single- and Dual-Polarized Frequency-Selective Rasorbers With Wide Absorption Bands Based on Stepped Impedance Resonator," IEEE Access, vol. 9, pp. 22317-22327, 2021.

[24] Mário Silveirinha and Nader Engheta, "Tunneling of Electromagnetic Energy through Subwavelength Channels and Bends using $\varepsilon$-Near-Zero Materials," Phys. Rev. Lett. vol. 97, pp. 157403, Oct. 2006.

[25] M. Mitrovic, B. Jokanovic, and N. Vojnovic, "Wideband tuning of the tunneling frequency in a narrowed epsilon-near-zero channel," IEEE Antennas Wireless Propag. Lett., vol. 12, pp. 631-634, May 2013.
[26] B. Edwards, A. Alu, M. E. Young, M. Silveirinha, and N. Engheta, "Experimental verification of epsilon-near-zero metamaterial coupling and energy squeezing using a microwave waveguide," Phys. Rev. Lett., vol. 100, no. 3, pp. 033903, 2008.

[27] N. Vojnovic, B. Jokanovic, M. Radovanovic, F. Medina and F. Mesa, "Modeling of Nonresonant Longitudinal and Inclined Slots for Resonance Tuning in ENZ Waveguide Structures," IEEE Trans. Antennas Propag., vol. 63, no. 11, pp. 5107-5113, Nov. 2015.

[28] Y. Liu, F. Sun, Y. Yang, Z. Chen, J. Zhang, S. He and Y. Ma, "Broadband Electromagnetic Wave Tunneling with Transmuted Material Singularity," Phys. Rev. Lett., vol 125, pp. 207401, Nov. 2020.

[29] S. Zhong, Y. Ma, and S. He, "Perfect absorption in ultrathin anisotropic $\varepsilon$-near-zero metamaterials,"Appl. Phys. Lett. vol.105, pp. 023504, 2014.

[30] S. Zhong, T. Liu, J. Huang, and Y. Ma, "Giant power enhancement for quasi-omnidirectional light radiation via $\varepsilon$-near-zero materials," Opt. Express, vol. 26, no. 3, pp. 2231-2241, Feb. 2018

[31] Y. He, Y. Li, Z. Zhou et al., "Wideband Epsilon-Near-Zero Supercoupling Control through Substrate-Integrated Impedance Surface," Adv. Theory Simul., vol. 2, pp.1900059, May 2019.

[32] M. Guglielmi and A. A. Oliner, "Multimode network description of a planar periodic metal-strip grating at a dielectric interface. I. Rigorous network formulations," IEEE Trans. Microw. Theory Techn., vol. 37, no. 3, pp. 534-541, March 1989.

[33] Samwha Electronics. Ferrite Absorber. Accessed: Feb. 8, 2020. [Online]. Available:http://www.samwha.com/electronics/news/news_product3.as px

[34] B. Li and Z. Shen, "Dual-Band Bandpass Frequency-Selective Structures With Arbitrary Band Ratios," IEEE Trans. Antennas Propag., vol. 62, no. 11, pp. 5504-5512, Nov. 2014

[35] A. A. Omar and Z. Shen, "Thin Bandstop Frequency-Selective Structures Based on Loop Resonator," IEEE Trans. Microw. Theory Techn., vol. 65, no. 7, pp. 2298-2309, Jul. 2017. 